\title{
Factorization Hack of RSA Secret Numbers
}

\author{
Andysah Putera Utama Siahaan \\ Faculty of Computer Science \\ Universitas Pembangunan Panca Budi \\ Jl. Jend. Gatot Subroto Km. 4,5 Sei Sikambing, 20122, Medan, Sumatera Utara, Indonesia
}

\begin{abstract}
RSA always uses two big prime numbers to deal with the encryption process. The public key is obtained from the multiplication of both figures. However, we can break it by doing factorization to split the public key into two individual numbers. Cryptanalysis can perform the public key crack by knowing its value. The private key will be soon constructed after the two numbers retrieved. The public key is noted as " $N$ ", while " $N=P$. Q". This technique is unclassified anymore to solve the RSA public and private key. If it is successfully factored into $p$ and $q$ then $\Phi(N)=(P-1)$. $(Q-1)$ can be further calculated. By having the public key e, the private key $d$ will be solved. Factorization method is the best way to do the demolition. This study concerns to numbers factorization. GCD calculation will produce the encryption " $E$ " and decryption " $D$ " keys, but it depends on the computer speed.
\end{abstract}

Keywords - Cryptography, RSA, Public Key, Factorization

\section{INTRODUCTION}

There are various techniques should be used to protect the confidential image data from unauthorized accessand there are many to breach the security as well[1]. RSA is a public key cryptographic algorithm that works on two main cryptographic processes, public and private key [6].The power of RSA is in the prime numbers. The longer the key is used, the longer the time used to factor the public key. The main strength of the RSA algorithm is based on the difficulty of factoring large numbers into prime factors [3]. Although RSA is still difficult to solve, it has a security hole by simply knowing the ciphertext and public key. One of the techniques is to attack the RSA public key by factoring the "N". This weakness is utilized to carry out attacks to test the security level of this algorithm. The most common attack on RSA is the factorization problem of handling enormous number. If there is a new rapid method has been developed, it is possible to dismantle the RSA. In 2005, the largest number factorization was commonly used throughout the 663 bits, using advanced distribution methods. RSA keys in general throughout 1024 through 2048 bits. Some experts believe that 1024-bit keys will be solved shortly, but 2048-bit keys are still difficult to solve in the future.Since RSA is breakable by factoring the "N", the security of RSA is often based on the integer factorization problem [2].According to mathematical theory, it is easy to get two big prime numbers. However, the factorization is not easy as we think [5]. We try to find the value of "P" and "Q" form the known public key, but we limit the length of the " $\mathrm{N}$ " value according to the computer clock speed.

\section{BASIC CONCEPT}

The symmetric key is one of the cryptographic systems that uses the same kind of keys for encryption and decryption while the asymmetric is where the encryption and decryption use the different keys [4]. The RSA algorithm is described by three people from MIT (Massachusetts Institute of Technology). There are Ron Rivest, Adi Shamir, and Len Adleman in 1977. The encryption and description processes on RSA come from the concept of prime numbers, and modulo arithmetic. The encryption and decryption keys are both integers. The encryption key is unclassified while the decrypt key is confidential. The decryption key is generated from several pieces of prime numbers together with the encryption key. This algorithm patented by MIT in 1983 in the United States as US Patent 4405829. This patent is valid until September $21^{\text {st }}, 2000$.

RSA has several attributes indicate the input and output parameters. There are seven aspects we have to understand before trying to hack the RSA secret key such as:

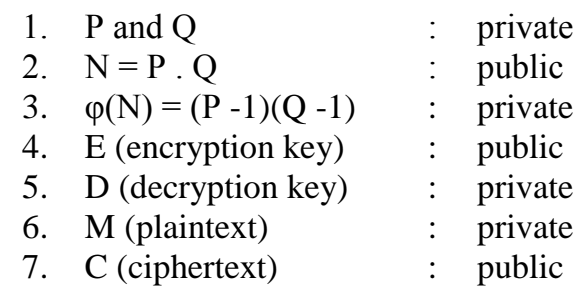

There are two signs above, public and private. Public means the value is unclassified. Everybody can get the information. However, the private means it is confidential. We must save it secretly. We hope nobody will find those value. The " $N$ " value is obtained from the multiplication "P" and "Q". Euler function is a function that is used in mathematical calculations on the RSA algorithm. Euler function is a function that is used in mathematical calculations on the RSA algorithm. Euler function defines $\phi(\mathrm{N})$ for $\mathrm{N} \geq 1$ indicates the number of positive integers $<\mathrm{N}$ 
relatively prime to $\mathrm{N}$. Two numbers "A" and "B" are relatively prime if $\operatorname{GCD}(\mathrm{A}, \mathrm{B})=1$. The value of " $\mathrm{E}$ " and " $\mathrm{D}$ " are used for both encryption and decryption respectively. The " $\mathrm{M}$ " and " $\mathrm{C}$ " are the plaintext and ciphertext byte arrays.

\section{Evaluation}

Implementation of RSA describes how the algorithm performs the message concealment process until the message is hidden. There are three most important stages of manipulating the message in the application of the RSA algorithm, such as the key generation process, the process of concealment of random messages (encryption) and the process of restoring the random messages into the initial message before manipulation (decryption). To hack the RSA secret key, we can simulate from the first step, that is the key generation process. After the generator produces it, the computer will note the values written on screen.

Table 1. Example of Key Generation

\begin{tabular}{|l|r|}
\hline Variable & \multicolumn{1}{|c|}{ Value } \\
\hline $\mathrm{P}$ & 5011 \\
\hline $\mathrm{Q}$ & 1093 \\
\hline $\mathrm{N}$ & 5477023 \\
\hline$\varphi$ & 5470920 \\
\hline
\end{tabular}

Table 1 illustrates the form of the key generation. The computer produces the random numbers for both "P" and " $Q$ ". Then " $N$ " and " $\varphi$ " are calculated by the earlier formula. The encryption and decryption keys can be generated by both "N" and " $\varphi$ ". Then we carry out the GCD between the " $\varphi$ "and new incremented variable "E". Not all the variable works. The test of $\operatorname{GCD}(\mathrm{E}, \varphi)=1$ must be done. The value which GCD $\neq 1$ cannot be accepted. We check the "E" from 2 to 30 and the result is that we have five possibilities, such as $11,17,19,23$, and 29. Sometimes, it is hard to guess which value is used by the sender since there are many probabilities of the prime numbers from 2 to $\varphi$. In this example, we use $\varphi=540920$. There are many prime numbers from 2 until 540920. However, it will be discovered. It depends on the computer speed. In this work, we assume that the value of the encryption used is 11 . Tabel 2 shows the selected values which GCD is 1 .

Table 2. The Encryption Key and GCD

\begin{tabular}{|c|c|}
\hline $\mathbf{E}$ & GCD \\
\hline 2 & 2 \\
\hline 3 & 3 \\
\hline 4 & 4 \\
\hline 5 & 5 \\
\hline 6 & 6 \\
\hline 7 & 7 \\
\hline
\end{tabular}

\begin{tabular}{|c|c|}
\hline 8 & 8 \\
\hline 9 & 9 \\
\hline 10 & 10 \\
\hline 11 & 1 \\
\hline 12 & 12 \\
\hline 13 & 13 \\
\hline 14 & 14 \\
\hline 15 & 15 \\
\hline 16 & 8 \\
\hline 17 & 1 \\
\hline 18 & 18 \\
\hline 19 & 1 \\
\hline 20 & 20 \\
\hline 21 & 21 \\
\hline 22 & 2 \\
\hline 23 & 1 \\
\hline 24 & 24 \\
\hline 25 & 5 \\
\hline 26 & 26 \\
\hline 27 & 9 \\
\hline 28 & 28 \\
\hline 29 & 1 \\
\hline 30 & 30 \\
\hline & \\
\hline
\end{tabular}

Soon after the encryption key is obtained, the decryption key can be processed by doing the following formula.

$$
\begin{gathered}
D=\frac{\varphi \cdot \mathrm{K}+1}{E} \\
R=(\varphi \cdot \mathrm{K}+1) \operatorname{Mod} E
\end{gathered}
$$

The first formula is to find the " $D$ " (encryption value). But not all the value retrieved can be used. The second is Rest, " $R$ " is a determinant whether the value of " $D$ " works. Looping is very important to search for the " $R$ " return zero value. Since R has more than zero, " $\mathrm{D}$ " will not work as the decryption key.

Table 3. The decryption key

\begin{tabular}{|c|c|c|}
\hline K & D & Rest \\
\hline 2 & 994712,82 & 9 \\
\hline 3 & 1492069,2 & 2 \\
\hline 4 & 1989425,5 & 6 \\
\hline 5 & 2486781,9 & 10 \\
\hline 6 & 2984138,3 & 3 \\
\hline 7 & 3481494,6 & 7 \\
\hline 8 & 3978851 & 0 \\
\hline 9 & 4476207,4 & 4 \\
\hline 10 & 4973563,7 & 8 \\
\hline 11 & 5470920,1 & 1 \\
\hline 12 & 5968276,5 & 5 \\
\hline
\end{tabular}




\begin{tabular}{|c|c|c|}
\hline 13 & 6465632,8 & 9 \\
\hline 14 & 6962989,2 & 2 \\
\hline 15 & 7460345,5 & 6 \\
\hline 16 & 7957701,9 & 10 \\
\hline 17 & 8455058,3 & 3 \\
\hline 18 & 8952414,6 & 7 \\
\hline 19 & 9449771 & 0 \\
\hline 20 & 9947127,4 & 4 \\
\hline 21 & 10444484 & 8 \\
\hline 22 & 10941840 & 1 \\
\hline 23 & 11439196 & 5 \\
\hline 24 & 11936553 & 9 \\
\hline 25 & 12433909 & 2 \\
\hline 26 & 12931266 & 6 \\
\hline 27 & 13428622 & 10 \\
\hline 28 & 13925978 & 3 \\
\hline 29 & 14423335 & 7 \\
\hline 30 & 14920691 & 0 \\
\hline
\end{tabular}

Table 3 shows there are 29 sample of "K", "D" and "Rest". There are only three datas that states zero value, such as data 8,19 and 30 . But, in this case, the correct decryption key for the encryption key 11 as discussed before is the first discovery, that is $\mathrm{K}=8$ and $\mathrm{D}=$ 3978851.In Table 4 , all parameters can directly implemented to plaintext and ciphertext.

Table 4. The complete parameters of RSA

\begin{tabular}{|l|r|}
\hline Variable & \multicolumn{1}{|c|}{ Value } \\
\hline $\mathrm{P}$ & 5011 \\
\hline $\mathrm{Q}$ & 1093 \\
\hline $\mathrm{N}$ & 5477023 \\
\hline $\mathrm{T}$ & 5470920 \\
\hline $\mathrm{E}$ & 11 \\
\hline $\mathrm{D}$ & 3978851 \\
\hline
\end{tabular}

That was the complete story of RSA. In this section, we try to break the $\mathrm{N}=5477023$. By factorizing it, we will obtain the "P" and "Q" simultenaously. First we calculate the SQRT(N) and test the value is square root or not.

$$
\begin{aligned}
\mathrm{A} \quad & \mathrm{SQRT}(\mathrm{N}) \\
& =\operatorname{SQRT}(5477023) \\
& =2340.304 \quad \text { (must be rounded }
\end{aligned}
$$

down)

$$
=2340
$$

Since $A$. A $\neq N, 5475600 \neq 5477023$, we add $A$ to 1 . The value of "A" will be 2341

$$
\begin{aligned}
\mathrm{A} & =\mathrm{A}+1 \\
& =2340+1 \\
& =2341 \\
\mathrm{~B} & =\mathrm{A} \cdot \mathrm{A}-\mathrm{N}
\end{aligned}
$$

$$
\begin{aligned}
& =2341 \cdot 2341-5477023 \\
& =3258 \\
& =\sqrt{B} \\
& =\sqrt{3258} \\
& =57 \\
& =\mathrm{C} \cdot \mathrm{C} \\
& =57.57 \\
& =3249 \\
& =\mathrm{B}-\mathrm{D} \\
& =3258-3249 \\
& =9
\end{aligned}
$$$$
\mathrm{C} \quad=\sqrt{B}
$$$$
\mathrm{D} \quad=\mathrm{C} \cdot \mathrm{C}
$$$$
\mathrm{R}=\mathrm{B}-\mathrm{D}
$$

The variable " $R$ " is used to determined the two prime numbers. " $R$ " means Rest. The progress is looped until the $\mathrm{R}=0$ is achieved. Since " $\mathrm{R}$ " does not produce zero, the " $\mathrm{A}$ " and " $\mathrm{B}$ " are malfunctioned. In Table 5 below, the complete progress of these five parameters cycle.

Table 5. The cycle of "A", "B", "C", "D" and "R" (part 1)

\begin{tabular}{|r|r|r|r|r|r|}
\hline Cycle & A & \multicolumn{1}{c|}{ B } & \multicolumn{1}{c|}{ C } & \multicolumn{1}{c|}{ D } & \multicolumn{1}{c|}{ R } \\
\hline 1 & 2341 & 3258 & 57 & 3249 & 9 \\
\hline 2 & 2342 & 7941 & 89 & 7921 & 20 \\
\hline 3 & 2343 & 12626 & 112 & 12544 & 82 \\
\hline 4 & 2344 & 17313 & 131 & 17161 & 152 \\
\hline 5 & 2345 & 22002 & 148 & 21904 & 98 \\
\hline 6 & 2346 & 26693 & 163 & 26569 & 124 \\
\hline 7 & 2347 & 31386 & 177 & 31329 & 57 \\
\hline 8 & 2348 & 36081 & 189 & 35721 & 360 \\
\hline 9 & 2349 & 40778 & 201 & 40401 & 377 \\
\hline 10 & 2350 & 45477 & 213 & 45369 & 108 \\
\hline 11 & 2351 & 50178 & 224 & 50176 & 2 \\
\hline 12 & 2352 & 54881 & 234 & 54756 & 125 \\
\hline 13 & 2353 & 59586 & 244 & 59536 & 50 \\
\hline 14 & 2354 & 64293 & 253 & 64009 & 284 \\
\hline 15 & 2355 & 69002 & 262 & 68644 & 358 \\
\hline 16 & 2356 & 73713 & 271 & 73441 & 272 \\
\hline 17 & 2357 & 78426 & 280 & 78400 & 26 \\
\hline 18 & 2358 & 83141 & 288 & 82944 & 197 \\
\hline 19 & 2359 & 87858 & 296 & 87616 & 242 \\
\hline 20 & 2360 & 92577 & 304 & 92416 & 161 \\
\hline 21 & 2361 & 97298 & 311 & 96721 & 577 \\
\hline 22 & 2362 & 102021 & 319 & 101761 & 260 \\
\hline 23 & 2363 & 106746 & 326 & 106276 & 470 \\
\hline 24 & 2364 & 111473 & 333 & 110889 & 584 \\
\hline 25 & 2365 & 116202 & 340 & 115600 & 602 \\
\hline 26 & 2366 & 120933 & 347 & 120409 & 524 \\
\hline 27 & 2367 & 125666 & 354 & 125316 & 350 \\
\hline 28 & 2368 & 130401 & 361 & 130321 & 80 \\
\hline 29 & 2369 & 135138 & 367 & 134689 & 449 \\
\hline 30 & 2370 & 139877 & 374 & 139876 & 1 \\
\hline
\end{tabular}


Let's focus on the "R" value. We have done the calculation for 30 times. However, the "R" still return the wrong value. The progress must be looped until it shows zero.

Table 6. The cycle of "A", "B", "C", "D" and "R" (part 2)

\begin{tabular}{|r|l|c|c|c|r|}
\hline Cycle & A & B & C & D & \multicolumn{1}{|c|}{$\mathbf{R}$} \\
\hline 683 & 3023 & 3661506 & 1913 & 3659569 & 1937 \\
\hline 684 & 3024 & 3667553 & 1915 & 3667225 & 328 \\
\hline 685 & 3025 & 3673602 & 1916 & 3671056 & 2546 \\
\hline 686 & 3026 & 3679653 & 1918 & 3678724 & 929 \\
\hline 687 & 3027 & 3685706 & 1919 & 3682561 & 3145 \\
\hline 688 & 3028 & 3691761 & 1921 & 3690241 & 1520 \\
\hline 689 & 3029 & 3697818 & 1922 & 3694084 & 3734 \\
\hline 690 & 3030 & 3703877 & 1924 & 3701776 & 2101 \\
\hline 691 & 3031 & 3709938 & 1926 & 3709476 & 462 \\
\hline 692 & 3032 & 3716001 & 1927 & 3713329 & 2672 \\
\hline 693 & 3033 & 3722066 & 1929 & 3721041 & 1025 \\
\hline 694 & 3034 & 3728133 & 1930 & 3724900 & 3233 \\
\hline 695 & 3035 & 3734202 & 1932 & 3732624 & 1578 \\
\hline 696 & 3036 & 3740273 & 1933 & 3736489 & 3784 \\
\hline 697 & 3037 & 3746346 & 1935 & 3744225 & 2121 \\
\hline 698 & 3038 & 3752421 & 1937 & 3751969 & 452 \\
\hline 699 & 3039 & 3758498 & 1938 & 3755844 & 2654 \\
\hline 700 & 3040 & 3764577 & 1940 & 3763600 & 977 \\
\hline 701 & 3041 & 3770658 & 1941 & 3767481 & 3177 \\
\hline 702 & 3042 & 3776741 & 1943 & 3775249 & 1492 \\
\hline 703 & 3043 & 3782826 & 1944 & 3779136 & 3690 \\
\hline 704 & 3044 & 3788913 & 1946 & 3786916 & 1997 \\
\hline 705 & 3045 & 3795002 & 1948 & 3794704 & 298 \\
\hline 706 & 3046 & 3801093 & 1949 & 3798601 & 2492 \\
\hline 707 & 3047 & 3807186 & 1951 & 3806401 & 785 \\
\hline 708 & 3048 & 3813281 & 1952 & 3810304 & 2977 \\
\hline 709 & 3049 & 3819378 & 1954 & 3818116 & 1262 \\
\hline 710 & 3050 & 3825477 & 1955 & 3822025 & 3452 \\
\hline 711 & 3051 & 3831578 & 1957 & 3829849 & 1729 \\
\hline 712 & 3052 & 3837681 & 1959 & 3837681 & 0 \\
\hline
\end{tabular}

The calculation continues until reach the desired value. Table 6 is the last cycle before reaching the zero value. After cycle 712 the "R" value gave the right value. $\mathrm{R}=0$ is what the factorization needs. The "P" and "Q" can be obtained then.

$$
\begin{array}{ll}
\mathrm{P} & =A-\sqrt{B} \\
& =3052-\sqrt{3837681} \\
& =3052-1959 \\
& =1093 \\
\mathrm{Q} & \\
& =3+\sqrt{B} \\
& =3052+\sqrt{3837681} \\
& =3052+1959
\end{array}
$$

$$
=5011
$$

Now we see the $\mathrm{P}=1093$ and $\mathrm{Q}=5011$, and the values before factorization are $\mathrm{P}=5011$ and $\mathrm{Q}=1039$. It does not matter because the "P" and "Q" are swappable.After we get those values, the process of finding "E" and "D" can be continued as discussed before.

\section{Conclusion}

There are many algorithms for symmetric and asymmetric can be used for encryption, decryption, key exchange and digital signature. To resolve the prime factors of RSA, we can use integer factorization algorithm. The RSA breach can be resolved easily by doing factorization on the public key. Everyone has already known the issue, and it is not a secret thing anymore. The speed depends on the public key length. The value of "P" and "Q" are not confidential since we use the small integer number. To protect them, we should use the big integer. There is no limitation for the integer. We can increase as long as the computer can calculate the formula properly.

\section{REFERENCES}

[1] A. P. U. Siahaan, "RC4 Technique in Visual Cryptography," SNATIA, Semarang, 2016.

[2] B. R. Ambedkar dan S. S. Bedi, "A New Factorization Method to Factorize RSA Public Key Encryption," International Journal of Computer Science, vol. 8, no. 6, pp. 242-247, 2011.

[3] H.-M. Sun, M.-E. Wu, W.-C. Ting dan M. J. Hinek, "Dual RSA and Its Security Analysis," IEEE Transactions on Information Theory, vol. 53, no. 8, 2007.

[4] A. P. U. Siahaan, "Three-Pass Protocol Concept in Hill Cipher Encryption Technique," SNATI, Yogyakarta, 2016.

[5] N. Hegde dan P. Deepthi, "Pollard RHO Algorithm for Integer Factorization and Discrete Logarithm Problem," International Journal of Computer Applications, vol. 121, no. 18, pp. 14-17, 2012.

[6] F. Nizar, F. Latheef dan A. Jamal, "RSA Based Encrypted Data Embedding Using APPM," International Journal of Engineering Trends and Technology, vol. 9, no. 15, pp. 777782, 2014 